\title{
Distributed Multi-Target Search and Track Assignment Using Consensus-Based Coordination
}

\author{
Tracie A. Severson* and Derek A. Paley ${ }^{\dagger}$, ${ }^{*}$ Department of Aerospace Engineering, University of Maryland, \\ College Park, MD 20742, ${ }^{\dagger}$ Department of Aerospace Engineering and Institute for Systems Research, University \\ of Maryland, College Park, MD 20742
}

\begin{abstract}
This paper presents a distributed, consensus-based approach to optimize radar resource management for ballistic missile surveillance and tracking. Each radar determines their preferred radar-to-target assignment by balancing radar loading and minimizing total radar usage. A unique global radar-totarget assignment that is robust to resource estimation error is generated by a distributed consensus algorithm. Performance is validated via Monte Carlo simulations.
\end{abstract}

\section{INTRODUCTION}

Multifunction radar technology supports the simultaneous execution of search and track functions [1]. To facilitate the radar's ability to dynamically prioritize its search and track tasking, a radar resource manager must optimize the allocation of radar resources to each function. Existing resource management approaches for a single radar are addressed in [2], [3] to reduce target state uncertainty. For multiple shipboard radars, [4] balances the relative residual radar resource capacity to minimize target migrations from one ship's search sector to another ship's sector. However, it prevents a radar from tracking targets outside its own search sector, which may be beneficial in balancing the resource load.

This paper applies techniques from probabilistic optimization, graph theory, and consensus control to design a theoretically justified, distributed framework for the assignment of ballistic missile (BM) and air defense (AD) search and track radar resources that balances and minimizes resource usage while accounting for the resources required to reduce target uncertainty to a desired level to support engagements. Balancing the resource utilization prevents a single radar from using all of its available resources, unless the other radars are also at their maximum capacity, which is important if a majority of the targets are detected by a subset of sensors. Minimizing total resource usage allows the remaining resources to be applied to additional search or track functions. Radars reach agreement on the global optimal targetassignment by exchanging and updating their individual targetassignment solutions over a communication network in an iterative fashion that yields consensus on a single solution. Monte Carlo simulations demonstrate that the optimal radarto-target assignment solution allows for a greater number of targets to be detected and tracked while preserving adequate resources for $\mathrm{AD}$ search.

\section{RADAR MODEL}

Radar resource usage for search and track is modeled using the radar range equation [5. pp. 30-35]. Each ballistic target is modeled as a linear dynamical system. Target measurements are processed by a tracking filter that provides the target's state and uncertainty. Tracking resources include the resources required to reduce the target uncertainty to support engagements.

\section{A. Resource Allocation}

The initial resource allocation is partitioned into AD Search, AD Track, BM Search, BM Track, and Spare as shown in Fig. 1(a) As BM targets are detected and tracked, BM Track consumes Spare resources to track new targets and reduce the uncertainty of existing BM targets (designated BM Track-Engage). Once all Spare resources are consumed, as depicted in Fig. 1(b), BM Track and BM Track-Engage may consume additional resources initially reserved for AD Search as illustrated in Fig. 1(c) and the AD search sector is redefined.

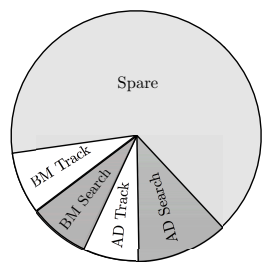

(a) Initial Allocation

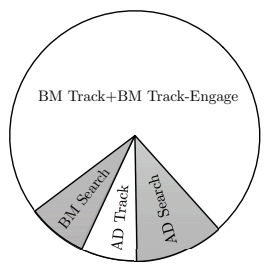

(b) Fixed Allocation

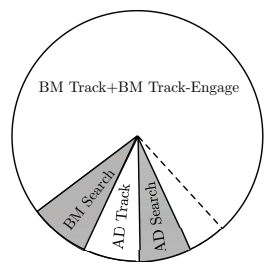

(c) Variable Allocation
Fig. 1. (a) Initial resource allocation prior to BM tracking; (b) resource allocation after BM track consumes spare resources; and (c) resource allocation after BM track resources consume spare and some AD search resources.

\section{B. Surveillance and Tracking Model}

The signal-to-noise ratio (SNR) is the standard measure of a radar's ability to detect a given target at a range $\rho$ from the radar. SNR is a linear function of the inverse search sector solid angle $\Omega$ and a quartic function of the inverse detection range $\rho[5$, pp. 30-90]:

$$
\mathrm{SNR}=\frac{P_{\text {avg }} A_{e} \sigma T_{\text {search }}}{4 \pi \kappa T_{0} L_{s} \rho^{4} \Omega}
$$

where $P_{a v g}$ is the average transmitted power, $A_{e}$ is the effective antenna area, $\sigma$ is the target radar cross section, $\kappa$ is Boltzmann's constant, $T_{0}$ is the radar system temperature, $L_{s}$ are the total system losses, and $T_{\text {search }}$ is the search scan time for solid angle $\Omega$ at range $\rho$. Radar resource usage is expressed in units of time by solving 11 for $T_{\text {search }}=a \rho^{4} \Omega$, where

\footnotetext{
${ }^{1}$ The solid angle subtended by a surface is the surface area of the portion of a unit sphere covered by the surface's radial projection onto the sphere [6].
} 
$a \triangleq 4 \pi \kappa T_{s}(S N R) L_{s} /\left(P_{a v g} A_{e} \sigma\right)$ is identical for all radars. For multiple search sectors, let $S_{k}$ represent the total number of $\mathrm{BM}$ and $\mathrm{AD}$ sectors for radar $k$, where $s \in\left\{1, \ldots, S_{k}\right\}$ is the search sector index. The total search scan time $T_{\text {search }, k}^{(t o t)}$ dedicated to searching all $S_{k}$ sectors of radar $k$ is expressed

$$
T_{\text {search }, k}^{(t o t)}=a \sum_{s=1}^{S_{k}} \rho_{s, k}^{4} \Omega_{s, k} .
$$

Let $X=\left[\begin{array}{llllll}x & y & z & \dot{x} & \dot{y} & \dot{z}\end{array}\right]^{T}$ be the target position and velocity and $X(n)$ be the state at time $n$, where the time step is $T=t(n+1)-t(n)$. The measurements collected by a radar are the range $\rho$, the azimuth $\theta$, and elevation $\phi$, subject to measurement noise with variance $\sigma_{\rho}^{2}, \sigma_{\theta}^{2}$, and $\sigma_{\phi}^{2}$, respectively. Radar measurements are transformed to Cartesian coordinates [7] so that the measurement equation is linear

$$
Z(n)=H(n) X(n)+V(n),
$$

where $Z=\left[\begin{array}{lll}x & y & z\end{array}\right]^{T}$ and $V \sim \mathcal{N}(0, R)$ is the Gaussian measurement error with covariance $R$ referred to in Cartesian coordinates.

One targets are detected, the array beam is steered directly to the target for track updates, thus

$$
\mathrm{SNR}=\frac{P_{t} G^{2} \lambda^{2} \sigma T_{\text {track }}}{(4 \pi)^{3} \kappa T_{0} L_{s} \rho^{4}},
$$

where $P_{t}$ is the peak transmitter power, $G$ is the antenna gain, $\lambda$ is the wavelength, and $T_{\text {track }}$ is the amount of time the target is in track per scan. Solving (4) for $T_{\text {track }}$ yields $T_{\text {track }}=c \rho^{4}$, where $c \triangleq(4 \pi)^{3} \kappa T_{0}(S N R) L_{s} /\left(P_{t} G^{2} \lambda^{2} \sigma\right)$. Let $M_{k}$ be the number of targets tracked by radar $k$ so that the total track time is

$$
T_{\text {track }, k}^{(\text {tot }}=c \sum_{m=1}^{M_{k}} \rho_{m, k}^{4},
$$

where $m=\left\{1, \ldots, M_{k}\right\}$ is the target index.

Successive measurement updates reduce the target state uncertainty to support an engagement. Motivated by [8], the projected reduction in target uncertainty for each radar-target pairing is found using the outputs of a minimum variance linear tracking filter that approximates the resources required to reduce target uncertainty to the engagement level.

Suppose radar $j$ is tracking target $m$ at time step $n$. Let $x_{m}(n)$ represent the target state estimate and $\Sigma_{m}^{j}(n)$ be the $6 \times 6$ error covariance. For each radar $k$ that can track target $m$, let $\Sigma_{m}^{k}(n+1)$ represent the error covariance matrix that results if tracking responsibilities for target $m$ switched to radar $k$. To account for the communication latency $T_{\text {com }}$, we have

$$
\Sigma_{m}^{k}(n)= \begin{cases}\Sigma_{m}^{j}(n) & \text { if } k=j \\ \left(\Phi\left(T_{\text {com }}\right)\right)^{T} \Sigma_{m}^{j}(n)\left(\Phi\left(T_{\text {com }}\right)\right) & \text { otherwise, }\end{cases}
$$

where $\Phi$ is the state transition matrix. Let $K_{m}^{k}$ be the projected estimate of the Kalman gain if radar $k$ were assigned tracking responsibilities for target $m$, given by $K_{m}^{k}(n+$ 1) $=\Sigma_{m}^{k}(n) H^{T}\left[H \Sigma_{m}^{k}(n) H^{T}+R_{m}^{k}(n+1)\right]^{-1}$. The projected state covariance for radar $k$ and target $m$ is

$$
\Sigma_{m}^{k}(n+1)=\left(I-K_{m}^{k}(n+1) H\right) \Sigma_{m}^{k}(n),
$$

where $I$ is the $6 \times 6$ identity matrix. The track uncertainty for each radar-to-target pairing is found by summing the diagonal position elements of (7) to obtain

$$
(T U)_{m}^{k}(n)=\operatorname{Tr}\left[\Phi^{T} \sum_{m}^{k}(n) \Phi\right] .
$$

The resources required to reach a desired engagement track uncertainty $(T U)_{e n g}$ are computed using a linear interpolation between the current target uncertainty and the projected uncertainty at the next time step

$$
T_{\text {eng }, k}^{m}=\frac{(T U)_{m}^{k}(n)-(T U)_{e n g}}{\Delta(T U)_{m}^{k}} T_{\text {track }, k},
$$

where $\Delta(T U)_{m}^{k}=(T U)_{m}^{k}(n)-(T U)_{m}^{k}(n+1)$. The total target engagement time is

$$
T_{\text {eng,k }}^{(t o t)}=c \sum_{m=1}^{M_{k}} T_{e n g, k}^{m} .
$$

Combining (2), (5), and (10) yields the total resources in units of time required to complete the desired search, track, and engagement requirements of radar $k$ for all $M_{k}$ targets. In what follows, let $P_{k} \triangleq\left(T_{\text {search }, k}^{(t o t)}+T_{\text {track }, k}^{(t o t)}+T_{\text {eng, }, k}^{(t o t)}+\epsilon_{k}\right) / T_{\text {max }}$ denote the total normalized resources used for search and track by radar $k$, where $\epsilon_{k}$ denotes the spare resources and $T_{\max }$ is the maximum available resource. The resource usage constraint is expressed $P_{k} \leq 1$.

\section{TARget Assignment Optimization}

The radar communication network is modeled as a strongly connected, balanced digraph $G_{\text {com }}$ with nodes $\mathcal{N}=\{1, \ldots, N\}$ and edges $\mathcal{E}_{\text {com }} \subseteq \mathcal{N} \times \mathcal{N}$. The edge set $\mathcal{E}_{\text {com }}$ contains all of the ordered (unweighted) pairs of directed communication links between nodes designated by $e_{j k} \triangleq(j, k)$. The entries $a_{k j}$ in the binary $N \times N$ adjacency matrix $A$ of $G_{c o m}$ are defined as $a_{k j}=1$ if $e_{j k} \in \mathcal{E}$ and $a_{k j}=0$ if $k=j$. The Laplacian matrix $L_{c o m}$ of $G_{c o m}$ is defined by entries $l_{k j}=-a_{k j}$ for $k \neq j$ and $l_{k k}=\sum_{l=1}^{N} a_{k j}[9]$. We also model the target-assignment network $G_{\text {track }}=\left(\mathcal{N} \cup \mathcal{M}, \mathcal{E}_{\text {track }}\right)$ as a bipartite graph where $\mathcal{N}=\{1, \ldots, N\}$ represents the set of radars, $\mathcal{M}=\{1, \ldots, M\}$ represents the set of targets, and $\varepsilon_{\text {track }} \subseteq \mathcal{M} \times \mathcal{N}$ is the unweighted set of all possible radarto-target pairings. A target-assignment solution is the set of radar-to-target pairings that assigns exactly one radar in the set $\mathcal{N}$ to each detected target in the disjoint set $\mathcal{M}$, represented by the adjacency matrix of the bipartite graph $G_{\text {track }}$. Fig. 2 illustrates one possible target-assignment solution $G_{\text {track }}$ for $M=6$ targets and $G_{c o m}$ for $N=4$ radars.

The framework of the target-assignment optimization problem is based upon meeting the following two objectives: (i) balancing radar resource tasking and (ii) minimizing total radar resource usage. Objectives (i) and (ii) are formulated using a quadratic form based on a weighted graph Laplacian that reflects the priorities of balancing resource usage versus total resource usage. The solution to the optimization problem is the target-assignment graph $G_{\text {track }}$ that best achieves the convex combination of both objectives. A genetic algorithm [10] is used to solve for the optimal target-assignment solution, 


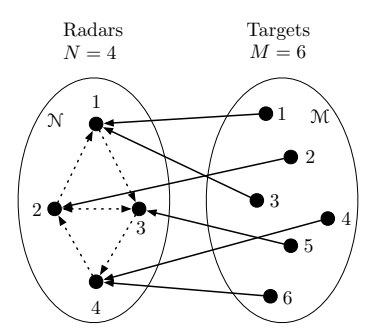

Fig. 2. (a) Radar communication network $G_{\text {com }}$ for $N=4$ radars and (b) target assignment network $G_{\text {track }}$ for $M=6$ targets.

because an exhaustive search would yield excessive computational time requirements. An initial population of targetassignment graphs is encoded as a binary solution as follows. Let $G_{\text {track, }}$ be the target-assignment solution proposed by radar $k$ for all radars and targets and let $A_{k} \in \mathbb{R}^{N \times M}$ be the adjacency matrix of $G_{t r a c k, k}$ such that

$$
a_{i j}= \begin{cases}1, & \text { if } G_{t r a c k, k} \text { assigns target } j \text { to radar } i \\ 0, & \text { otherwise. }\end{cases}
$$

Note that $A_{k}$ satisfies $\sum_{j=1}^{N} a_{j k}=1$ which represents the constraint that each target is assigned to exactly one radar and no (detected) targets are unassigned. Each local targetassignment solution is evaluated based on an objective (fitness) function described below.

Objective (i) of the target-assignment optimization is to balance radar resources over the radar communication network $G_{\text {com }}$, (i.e., to minimize the differences in radar usage between all pairs of communicating radars). Let $P=\left[\begin{array}{llll}P_{1} & P_{2} & \ldots & P_{N}\end{array}\right]^{T}$ be the collection of all normalized radar resource allocations. Let $L_{c o m}$ be the graph Laplacian of $G_{c o m}$. The optimal targetassignment network $G_{\text {track }}$ balances radar tasking across $G_{\text {com }}$ by minimizing the Laplacian quadratic form [9]

$$
P^{T} L_{c o m} P=\sum_{(j, k) \in \mathcal{E}_{c o m}}\left(P_{k}-P_{j}\right)^{2} .
$$

Objective (ii) of the target-assignment optimization problem is to minimize the total tracking resource usage over all radars in $G_{c o m}$. Let $I_{N}$ be the $N \times N$ identity matrix, which represents the Laplacian of a balanced graph consisting of only self-loops, i.e. $\left[l_{i j}\right]=2$ for all $i=j$, and $\left[l_{i j}\right]=0$ otherwise. Minimizing total (squared) radar resources is equivalent to minimizing the Laplacian quadratic form

$$
P^{T} P=P^{T} I_{N} P
$$

Objectives (i) and (ii) are combined into a single multiobjective cost function by first normalizing each objective function by its maximum value and then forming a combined quadratic form. Let $K, 0 \leq K \leq 1$ be a weighting factor that represents the priority of balancing as compared to minimizing total resource usage. The overall cost function is the convex combination

$J\left(G_{\text {track }} ; G_{c o m}\right) \triangleq P^{T}\left[K \frac{2}{N^{2}(N-1)} L_{c o m}+(1-K) \frac{1}{N} I_{N}\right] P$.

The target assignment solution that balances resource usage and minimizes total resources is

$$
G_{\text {track }}^{*}=\underset{G_{\text {track }}}{\operatorname{argmin}}\left(J\left(G_{\text {track }} ; G_{\text {com }}\right)\right) .
$$

Reaching consensus ensures exactly one common target assignment solution and avoids the possibility that a target is not tracked. The binary consensus algorithm [11] described here also provides robustness to errors in radar resource estimation, track uncertainty improvements, and communication noise.

Let $G_{\text {track,k }}$ be the target assignment solution proposed by radar $k$ using $\sqrt{15}$ and define $\bar{A}_{k} \in \mathbb{R}^{N M \times 1}$ to be the elements of the adjacency matrix of $G_{\text {track, },}$ in (11) taken column-wise and arranged as a single column. Let $q_{j k}(n) \in \mathbb{R}$ represent the zero-mean Gaussian receiver noise at the $n$th time step in the transmission of the target assignment solution from the $k$ th radar to the $j$ th one with constant variance $\sigma_{w}^{2}$. Let $\mathcal{N}_{j}$ represent the set of radars $k$ that can communicate with radar $j$ and $\left|\mathcal{N}_{j}\right|$ be the size of $\mathcal{N}_{j}$. Dec[·] denotes a decision function for the binary $0-1$ entry performed on each element such that $\operatorname{Dec}[x]=1$ if $x \geq 0.5$ and 0 otherwise. Each radar updates its own target assignment solution based on the received information as follows [11]:

$$
\bar{A}_{j}(n+1)=\operatorname{Dec}\left[\frac{\bar{A}_{j}(n)+\sum_{k \in \mathcal{N}_{j}}\left(\bar{A}_{k}(n)+q_{j k}(n)\right)}{2+\left|\mathcal{N}_{j}\right|}\right] .
$$

The update rate $(16)$ is the binary form of a discrete consensus algorithm [12].

\section{RADAR System Simulation}

Consider four identical radars with BM search sectors optimized about the predicted target trajectories. Target measurements are updated every $T=2$ seconds; radars optimize the target-assignment solution and reach consensus every 4 seconds with a communication latency of $T_{c o m}=6$ seconds. For all search sectors, a five-second search revisit rate is instructed. The engagement track uncertainty is $14.2 \mathrm{~km}^{2}$ [less than 0.15 seconds of error for a BM target traveling at 200 $\mathrm{km} / \mathrm{s}$ [13]]. Radar parameters used for this scenario are similar to those in [13].

The target launch rate $p(k)$ is modeled as a Poisson distribution $p(k)=\left(\lambda^{k} / k !\right) e^{\lambda}$, where $\lambda$ is the expected number of targets launched every $T$ seconds and $p(k)$ is the probability that $k=0,1,2, \ldots$ targets will be launched every $T$ seconds. Each target has maximum life span of 300 seconds, and vanishes on impact. Targets trajectories are chosen from a set of four launch points and two aim points with a larger percentage of the targets coming from the two launch points located near $(0,0)$, as shown in Fig. 3 Fig. 3 also plots the target aim points (denoted by the black X's) and the $2 \sigma$ ellipses of the impact point probability distribution. Targets are detected with a single-look probability of detection of 0.9 . Coordinated radars broadcast the target state and uncertainty over $G_{c o m}$, solve for optimal target-assignment using (15), and use this solution to initialize the binary consensus algorithm (16) with noise variance $\sigma_{w}^{2}=0.1$. Coordinated radars are compared with two uncoordinated alternatives: Independent radars and Shared radars. Independent radars only track targets detected within their own search sector. Shared radars may 


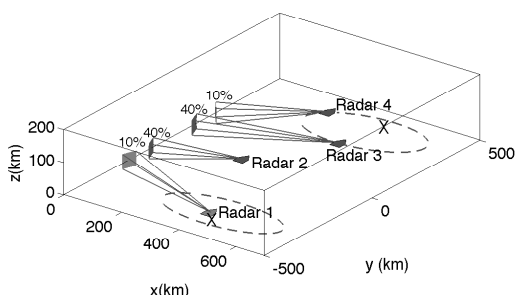

Fig. 3. (Radar locations and BM search sectors with respect to the target launch and impact point distributions.

track targets detected by a neighboring radar and multiple radars may track the same target. Figs. 4(a) 4(b) plot the

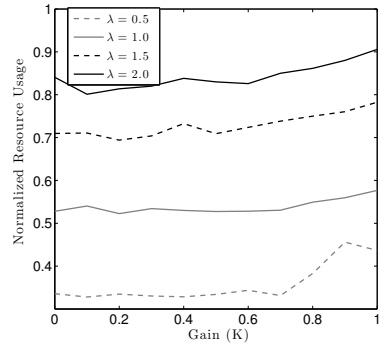

(a) Total resource

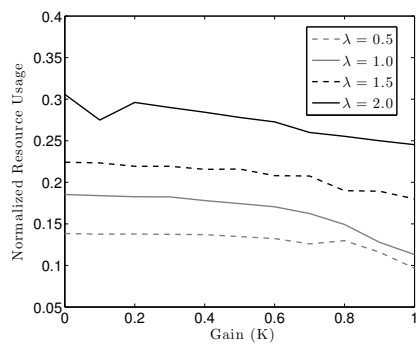

(b) Sum of pairwise difference
Fig. 4. Resource usage for all raid rates $\lambda$ depends on gain $K$.

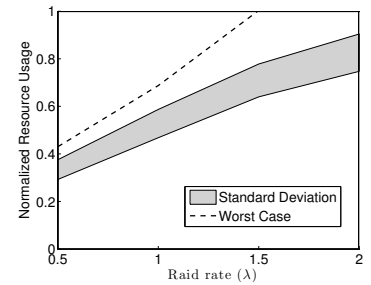

(a) Coordinated

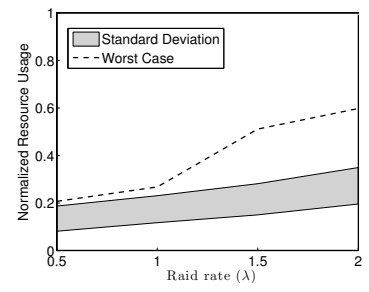

(c) Coordinated

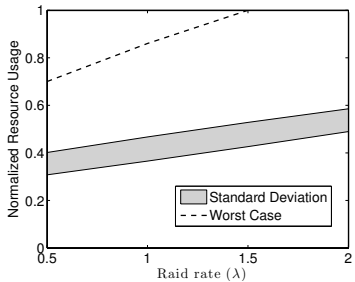

(b) Independent

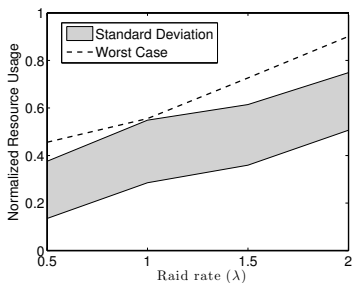

(d) Independent
Fig. 5. Total resource usage(a)-(b) and sum of pairwise difference in resource usage (c)-(d). Worse case radar usage for Coordinated radars is less than Independent and Shared radars (not shown) for all raid rates $\lambda$ and $K=0.5$.

maximum mean value of the total normalized radar resource usage and the mean of the sum of pairwise differences in resource usage for Coordinated radars over a range of launch rates $\lambda$ and gains $\mathrm{K}$. Note that smaller values of $\mathrm{K}$ result in less total resource usage as compared to larger values of $\mathrm{K}$, which result in a more balanced resource utilization as expected from 14. To highlight the benefit of coordination in terms of reducing the number of missed targets, Figs. 5(a)5(d) plot the mean-centered standard deviation (grey bar) and single radar worst-case (dotted-line) total resource usage and sum of pairwise differences in resource usage statistics from the Monte Carlo simulations for all raid rates and a fixed value $K=0.5$, for both Coordinated and Independent radars. (Shared radar performance is not shown since their maximum resource usage is equal to one for all raid rates.) While Independent radars are observed to use less resources, their resource usage is unbalanced due to a lack of coordination and leads to a greater number of missed targets as compared to Coordinated radars(Table I).

TABLE I

TOtAl Number OF Missed TARgets FOR $K=0.5$

\begin{tabular}{|c|c|c|c|c|}
\hline & $\lambda=0.5$ & $\lambda=1.0$ & $\lambda=1.5$ & $\lambda=2.0$ \\
\hline Coordinated & 0 & 0 & 1 & 14 \\
\hline Independent & 0 & 0 & 4 & 38 \\
\hline Shared & 11 & 51 & 79 & 94 \\
\hline
\end{tabular}

\section{CONCLUSION}

In this paper a distributed, consensus-based optimization approach to radar resource management for BM and AD search and track is presented. The radar communication network and target-assignment network is modeled using graph theory. A target-assignment optimization algorithm that uses a binary genetic algorithm to balance and minimize radar resource usage across the radar network is developed. Radar resources include the additional resources required to reduce track uncertainty. A consensus-based approach to reaching agreement on the optimal target-assignment is presented. Monte Carlo simulations demonstrate that the distributed target-assignment approach uses less total resources and remains more balanced in resource usage than uncoordinated alternatives, reducing the number of missed targets.

\section{REFERENCES}

[1] S. Sabatini and M. Tarantino, Multifunction Array Radar - System Design and Analysis. Artech House, 1994.

[2] S. Ghosh, J. Hansen, R. Rajkumar, and J. Lehoczky, "Integrated resource management and scheduling with multi-resource constraints," in Proc. of the 25th IEEE Int. RTSS, 2004, pp. 12-22.

[3] A. Charlish, K. Woodbridge, and H. Griffiths, "Multi-target tracking control using continuous double auction parameter selection," in Proc. 15th Int. Conf. on Information Fusion, 2012, pp. 1269-1276.

[4] P.-S. Kang and C.-G. Lee, "Coordinated search and track by multiple phased array radars," in 10th IEEE Real-Time and Embedded Technology and Applications Symposium., 2004, pp. 227-235.

[5] M. Skolnik, Introduction to Radar Systems, 3rd ed. McGraw-Hill, 2001.

[6] E. W. Weisstein. Solid angle. [Online]. Available: http://mathworld. wolfram.com/SolidAngle.html

[7] D. Lerro and Y. Bar-Shalom, "Tracking with debiased consistent converted measurements versus ekf," in IEEE Trans. Aero. Elec. Sys., 1993, pp. 1015-1022.

[8] H. Lambert and D. Sinno, "Bioinspired resource management for multiple-sensor target tracking systems," M.I.T., Tech. Rep., 2011.

[9] R. Saber and R. Murray, "Consensus problems in networks of agents with switching topology and time-delays," IEEE Trans. on Automatic Cont., vol. 49, Sept. 2004.

[10] C.M.Fonseca and P. Fleming, "Genetic algorithms for multiobjective optimization: Formulation, discussion and generalization," in Proc. 5th Int. Conf. Genetic Algorithms, 1993, pp. 416-423.

[11] Y. Yuan and Y. Mostofi, "Impact of link qualities and network topology on binary consensus," in Proc. 28th Amer. Contr. Conf., June 2009, pp. $1821-1826$

[12] R. Saber and R. Murray, "Consensus and cooperation in networked multi-agent systems," in Proc. IEEE, vol. 95, Jan 2007, pp. 215-223.

[13] C.Bacchus, D. Bedford, P. Dailey, S. Hill, I.Barford, J.Chung, R. Hazle, and M. Mihocka, "Digital array radar for ballistic missile defense and counter-stealth systems analysis and parameter tradeoff study," Naval Postgraduate School, Tech. Rep. SE-06-001, 2006. 